\title{
TRANSIENT MODEL AND BOUNDARY INVERSE PROBLEM
}

\author{
J. Havelka*, J. Sýkora**
}

\begin{abstract}
Identification of material parameters is being used in many fields ranging from geophysics, medical imaging, archaeology, material science to preservation of historical structures, etc. We focus on problems in civil engineering described by a transient heat equation, where the intervention into the structure might not be possible, hence our approach utilize only boundary measurements. We employ a Bayesian inference method to reconstruct both parameter fields, i.e. thermal conductivity and volumetric heat capacity. The spatial variability is approximated by randomfield which generally forms bases of the unknown fields and reduces the problem. The proposed approach is computationally verified for various loading scenarios, solver setups and material field distributions.
\end{abstract}

\section{Keywords: boundary inverse, transient heat transfer, random-field, finite element method}

\section{Introduction}

Parameter estimation is one of the key procedures in numerical modelling. Moreover having the ability to analyse a given structure without damaging or intervening it opens up a new possibilities in many areas saving both time and financial resources. Application of the inverse analysis using only boundary measurements first appeared in the 1930s in geophysics, see paper (Langer, 1933) and then expanded to many fields such as medicine, see papers (Calderón, 2006; Holder, 2004; Cheng et al., 1989), archaeology, see paper (Campana and Piro, 2008) and/or structure analysis, see papers (Bakirov et al., 2004a,b; Huang and Chin, 2000; Jones et al., 1995; Kolehmainen et al., 2008; Toivanen et al., 2014). As lack of the information can not be remedied by any mathematical treatment, see paper (Lanczos, 1996), one have to usually provide a mathematical model describing the underlying physical phenomena and a sufficient set of measurements. In our case the mathematical model is described by a transient heat equation and the set of measurements is represented by a climatic dataset to capture realistic boundary conditions. The material fields are approximated by random-fields which coefficients are being recovered using a Bayesian inference. From results it can be seen that our approach for such tasks is a suitable method for identification of material heterogeneity.

\section{Numerical model}

The physical phenomena is in our case sufficiently described by a following set of equations

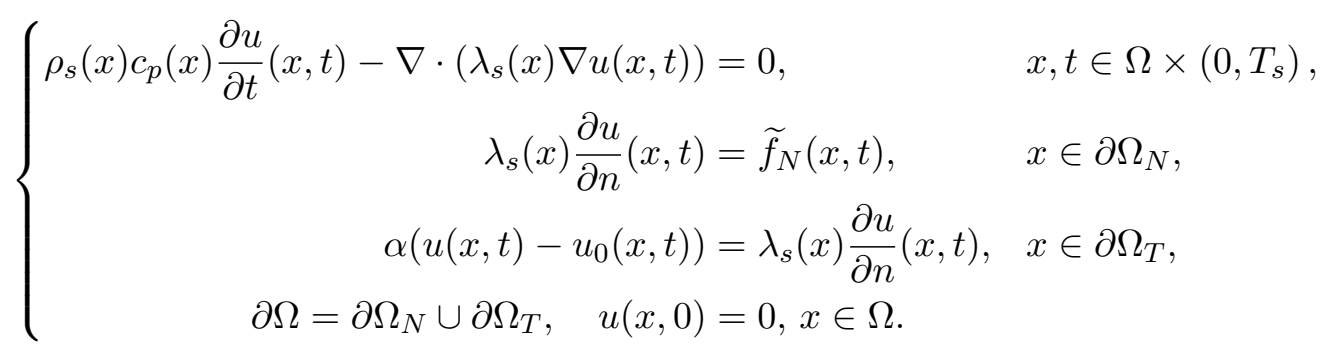

where $\rho_{s}$ is the volumetric mass density, $c_{p}$ is the specific heat capacity, $\lambda_{s}$ is the thermal conductivity coefficient and $T_{s}$ is the time when the simulation stops. The boundary of the domain is denoted by $\partial \Omega$

\footnotetext{
Ing. Jan Havelka: Department of Mechanics, Faculty of Civil Engineering, Czech Technical University in Prague, Thákurova 7/2077, 16629 Prague, CZ, jan.havelka.1@fsv.cvut.cz

** Ing. Jan Sýkora, PhD.: Department of Mechanics, Faculty of Civil Engineering, Czech Technical University in Prague, Thákurova 7/2077, 16629 Prague, CZ, jan.sykora.1@fsv.cvut.cz
} 
and the environmental factors are $u_{0}, \alpha$ and $\widetilde{f}_{N, T}$. The solution of such model is then obtained using finite element method for which the input parameters are environmental factors, boundary and initial conditions. The output of the model forms a vector $\mathbf{u} \in \mathbb{R}^{N_{n} N_{t}}$, where $N_{n}$ is the number of observed nodes located on the boundary and $N_{t}$ is the number of time steps.

In order to maintain the Neumann-to-Dirichlet sensing, i.e. to fulfil the basic uniqueness requirements by measuring Dirichlet data and prescribing Neumann loading conditions or vice versa, the set of equations in Eq. 1 is subjected to following constraint

Assumption 1 Let $\Gamma_{m}$ be a subset of boundary $\partial \Omega$ that is subjected to measurements. Then

$$
\Psi=\left(\partial \Omega_{N} \cup \partial \Omega_{T}\right):\left(\Psi \cap \Gamma_{m}\right) \notin \emptyset,
$$

must hold, i.e. the boundary subjected to measurements must contain at least some Neumann conditions.

Another set of constraints similar to the ones used in papers (Brown and Uhlmann, 1997; Sylvester and Uhlmann, 1986, 1987) is represented by following assumptions

Assumption 2 The material parameters $\lambda_{s}, \rho_{s}, c_{p}$ and the transfer coefficients $\alpha$ satisfy following

$$
\begin{array}{ll}
\text { (i) } & \lambda_{s} \in L^{\infty}(\Omega ; \mathbb{R}), \quad \inf _{x \in \Omega} \lambda_{s}(x)=\lambda_{s}^{+}>0, \\
\text { (ii) } & 0<\rho_{s}^{-} \leq \rho_{s} \leq \rho_{s}^{+}<\infty, \\
\text { (iii) } & 0<c_{p}^{-} \leq c_{p} \leq c_{p}^{+}<\infty, \\
\text { (iv) } & 0<\alpha^{-} \leq \alpha \leq \alpha^{+}<\infty,
\end{array}
$$

In the following numerical example, we employ realistic measurements of temperatures in the interior and exterior, see Fig. 1, which were used in boundary conditions (Eq. 2) with transfer coefficient $\alpha=10$.

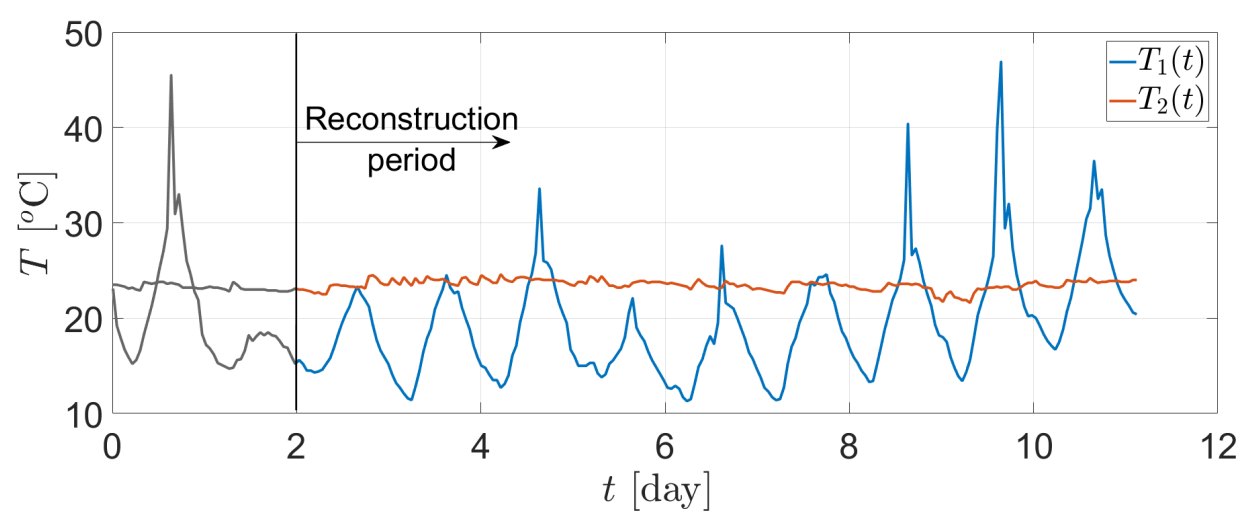

Fig. 1: Interior and exterior temperatures, data employed in the inverse process spans from day 2 to 11 .

$$
\begin{aligned}
& \left.\lambda_{s} \frac{\partial u}{\partial n}(x, t)\right|_{\partial \Omega_{1}}=10 \cdot\left(u(x, t)-T_{1}(t)\right), \\
& \left.\lambda_{s} \frac{\partial u}{\partial n}(x, t)\right|_{\partial \Omega_{2}}=10 \cdot\left(u(x, t)-T_{2}(t)\right) .
\end{aligned}
$$

A measurement of the observed part of boundary was conducted every 2 hours leading to $N_{t}=9 \cdot 24 / 2=$ 108 time steps. The measurement nodes $N_{n}=117$ are emphasized by asterisks in Fig. 2a and coincide with the boundaries $\partial \Omega_{1}$ and $\partial \Omega_{2}$ where the transfer boundary conditions (Eq. 2) are prescribed.

In contrast to the classical approach, where one have to put electrodes or thermocouples on a boundary in order to excite different boundary conditions and measure the system response, here we present an approach independent of such devices, relying only on a natural fluctuation of temperature. The system response after monitoring the object of interest for several days then has the potential to contain sufficient information about the underlying parameters. 
Each of the identified fields, i.e. $\lambda_{s}$ and $c_{v}$ are approximated by $m$ eigenvalues and eigenfunctions $\tilde{\phi}_{i}(\mathbf{x})=$ $\sqrt{\lambda_{i}} \phi_{i}(\mathbf{x})$ generated from Matérn covariance function with parameters $\nu=5$ and $\rho=0.25$ which has the following form

$$
C(d)=\sigma^{2} \frac{2^{1-\nu}}{\Gamma(\nu)}\left(\sqrt{2 \nu} \frac{d}{\rho}\right)^{\nu} K_{\nu}\left(\sqrt{2 \nu} \frac{d}{\rho}\right),
$$

where $\Gamma$ is the gamma function, $K_{\nu}$ is the modified Bessel function of the second kind, $\rho$ is the correlation length, $\nu$ relates to the differentiability and $d$ is the distance between two points. The covariance matrix for given domain discretisation is then decomposed into eigenvalues $\lambda_{i}$ and eigenmodes $\phi_{i}$ in a usual way. The individual fields are then approximated in the following way

$$
\kappa(\mathbf{x})=\exp \left(\sum_{i=0}^{m+1} \tilde{\phi}_{i}(\mathbf{x}) \xi_{i}\right) .
$$

The inverse procedure identifying the random variable coefficients $\xi_{i}$ is done in a standard fashion by sampling the posterior distribution and maximizing the likelihood, see papers (Kučerová et al., 2012). For each simulation we chose 75000 iterations of Markov Chain Monte Carlo (MCMC).

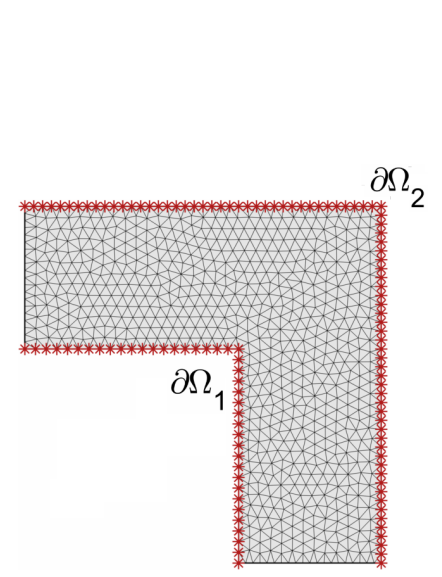

(a)

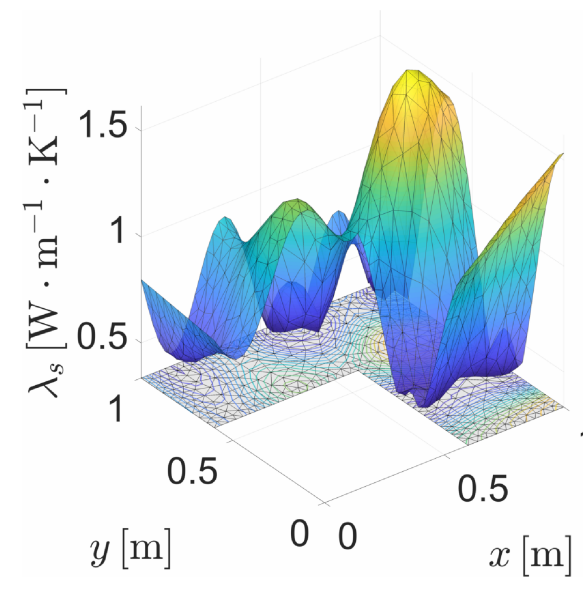

(b)

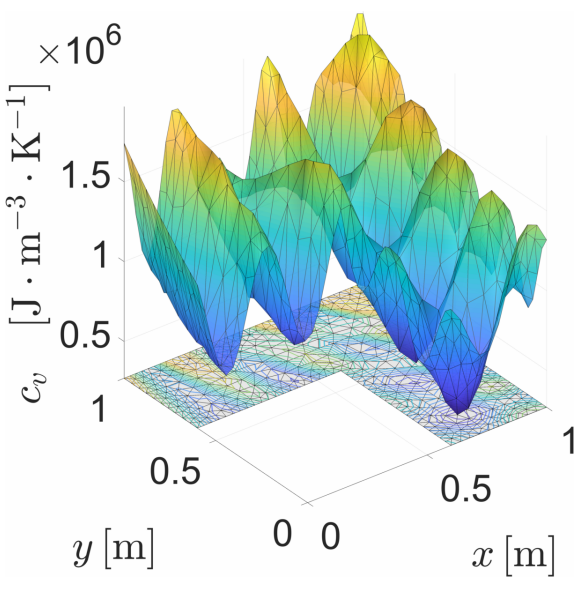

(c)

Fig. 2: (a) L-shaped domain with finite element discretisation utilised for the simulation and true material fields: (b) thermal conductivity, (c) volumetric heat capacity.

\section{Results}

The following study shows the evolution of an error depending on the number of eigenmodes used for each field. The errors are calculated as a mean value from last 5000 samples of MCMC as follows

$$
\varepsilon_{\kappa}=\frac{1}{5000} \sum_{i=70000}^{75000} \frac{\left\|\kappa_{i}-\kappa_{r}\right\|}{\left\|\kappa_{r}\right\|},
$$

where $\kappa_{i}$ is the reconstructed material field in the $i$-th iteration of MCMC and $\kappa_{r}$ is the reference field shown on Fig. 2b or Fig. 2c. The study is performed from 5 eigenmodes, which capture approximately $70 \%$ of the variance up to 50 eigenmodes, which capture $99.99 \%$ of the variance. The number of eigenmodes involved in the computation is shown on the horizontal axis in Fig. 3. One can notice overall higher error in reconstruction of volumetric capacity. This is caused by the choice of the correlation length $\rho$ which was chosen in favour of thermal conductivity which is much higher in comparison to the ideal correlation length for volumetric capacity, i.e. in our case $c_{v}$ has higher rate of fluctuations and hence smaller $\rho$, see Fig. $2 \mathrm{~b}$ and Fig. 2c.

\section{Conclusions}

It can be clearly seen that for given loading scenario, domain shape and material distribution the identified fields converge to the reference ones with the increasing number of eigenmodes. Since each iteration of MCMC require to evaluate the model response, our next intention is to accelerate the computation by using polynomial chaos to build a surrogate model which is much faster to evaluate. Another way to improve the calculation is to utilize one of adaptive MCMC methods and increase the convergence rate. 


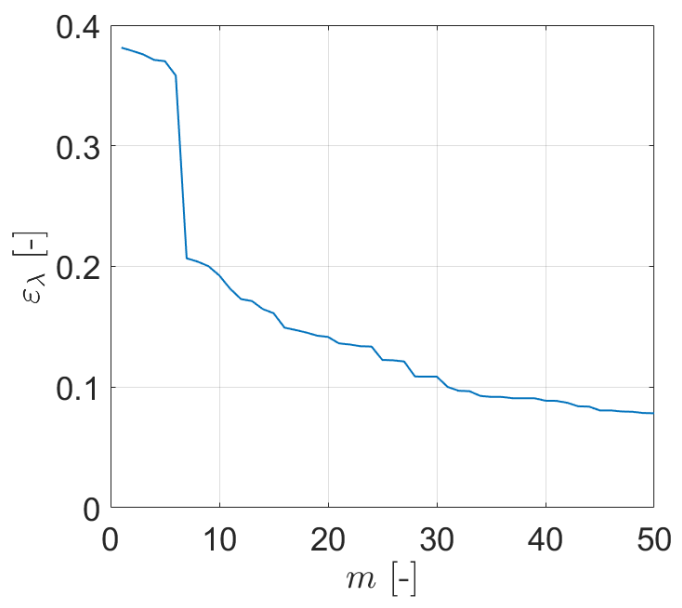

(a)

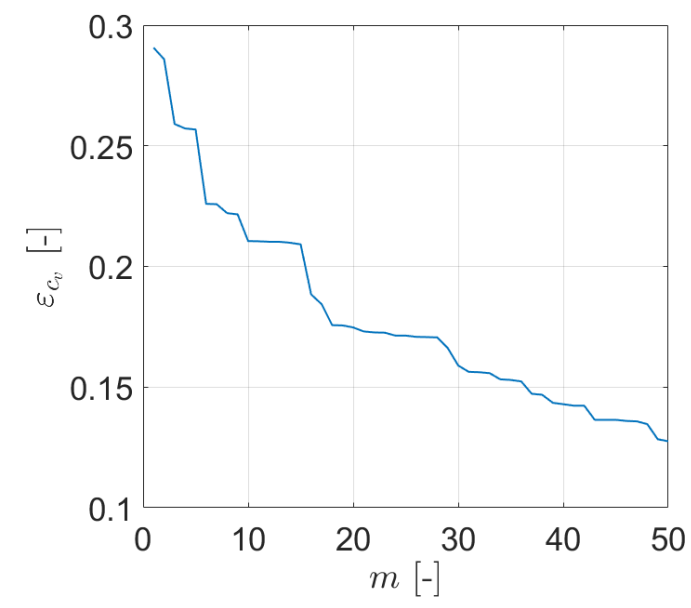

(b)

Fig. 3: (a) thermal conductivity error (b) volumetric capacity error.

\section{Acknowledgments}

The financial support of this research by the GA15-07299S and GA16-11473Y is gratefully acknowledged.

\section{References}

Bakirov, V. F., Kline, R. A., and Winfree, W. P. (2004), "A two-dimensional inverse problem in imaging the thermal conductivity of a non-homogeneous medium", AIP Conference Proceedings, 700(1), 469-476.

Bakirov, V. F., Kline, R. A., and Winfree, W. P. (2004), "Multiparameter thermal tomography", AIP Conference Proceedings, 700(1), 461-468.

Brown, R.M. and Uhlmann, G. A. (1997), "Uniqueness in the inverse conductivity problem for nonsmooth conductivities in two dimensions", Communications in Partial Differential Equations, 22(), 1009-1027.

Calderón, A. P. (2006), "On an inverse boundary value problem", Computational \& Applied Mathematics, 25, 133 138.

Campana, S. and Piro, S. (2008), Seeing the Unseen Geophysics and Landscape Archaeology, Taylor \& Francis.

Groetsch, Ch.W. (1993), Inverse Problems in the Mathematical Sciences, Springer.

Holder, D.S. (2004), Electrical Impedance Tomography: Methods, History and Applications, Taylor \& Francis.

Huang, C. and Chin, S. (2000), "A two-dimensional inverse problem in imaging the thermal conductivity of a nonhomogeneous medium", International Journal of Heat and Mass Transfer, 43(22), 4061-4071.

Hughes, T.J.R. (2012), The Finite Element Method: Linear Static and Dynamic Finite Element Analysis, Courier Corporation.

Cheng, K.S., Isaacson, D., Newell, J.C. and Gisser, D.G. (1989), "Electrode models for electric current computed tomography", IEEE Transactions on Biomedical Engineering, 36(9), 918-924.

Jones, M. R., Tezuka, A., and Yamada, Y. (1995), “Thermal tomographic detection of inhomogeneities", Journal of Heat Transfer, 117(4), 969-975.

Kirsch, A. (2011), An Introduction to the Mathematical Theory of Inverse Problems, Springer-Verlag New York.

Kolehmainen, V., Kaipio, J. P., and Orlande, H. R. B. (2008), "Reconstruction of thermal conductivity and heat capacity using a tomographic approach", International Journal of Heat and Mass Transfer, 51(7), 1866-1876.

Kučerová, A., Sýkora, J., Rosić, B., and Matthies, H. G. (2012), "Acceleration of uncertainty updating in the description of transport processes in heterogeneous materials", Journal of Computational and Applied Mathematics, 236(18), 4862-4872.

Lanczos, C. (1996), Linear Differential Operators, Society for Industrial and Applied Mathematics.

Langer, R.E. (1933), "An inverse problem in differential equations", Bulletin of the American Mathematical Society, 39(10), 814-820.

Sylvester, J. and Uhlmann, G. (1987), "A Global Uniqueness Theorem for an Inverse Boundary Value Problem", Annals of Mathematics, 125(1), 153-169.

Sylvester, J. and Uhlmann, G. (1986), "A uniqueness theorem for an inverse boundary value problem in electrical prospection", Communications on Pure and Applied Mathematics, 39(1), 91-112.

Toivanen, J. M., Tarvainen, T., Huttunen, J. M. J., Savolainen, T., Orlande, H. R. B, Kaipio, J. P., and Kolehmainen, V. (2014), "3D thermal tomography with experimental measurement data", International Journal of Heat and Mass Transfer, 78, 1126-1134. 